\title{
Enhanced luminescence of organic/metal nanostructure with symmetric dielectric layers for long-range surface plasmon polaritons
}

\author{
Nan-Fu Chiu ${ }^{1,2}$, Shou-Yu Nien ${ }^{3}$, Ling-Chih Chien ${ }^{2}$, Jiun-Haw Lee ${ }^{3}$, Chieh-Hsiung Kuan ${ }^{4}$, \\ Chii-Wann $\operatorname{Lin}^{1,2^{*}}$ \\ ${ }^{1}$ Institute of Electrical Engineering, ${ }^{2}$ Institute of Biomedical Engineering, \\ ${ }^{3}$ Graduate Institute of Electro-Optical Engineering, ${ }^{4}$ Graduate Institute of Electronics Engineering \\ National Taiwan University, No. 1, Sec. 4, Roosevelt Road, Taipei, Taiwan, Republic of China
}

\begin{abstract}
This paper is intended to demonstrate the effect of coupled long-range surface plasmon polaritons (LRSPPs) on the optical response of a lamellar grating nanostructure with organic material on the surface. This phenomenon gives rise to a selective spectral response and a local field enhancement which can be used in the context of nano-optics. This novel structure of nanofabricated device, consisting of coupled organic/metal nanostructure with specific width and symmetric dielectric structure. By designing the size and shape of the grating nanostructure, and the location of the organic Alq3 relative to the surfaces, Alq3 can be quenched, display increases in emission quantum yield, and decreasing the lifetimes. The combinations of organic/metal interface LRSPP mode can emit specific direction rather than isotropic emission. We discuss recent experimental results and potential applications in biosensor, organic light emitting diodes (OLEDs), polymer laser and organic solar cells of organic/metal grating enhanced emission resonance energy interactions.
\end{abstract}

Keywords: Long-range surface plasmon resonance, biosensor, organic light emitting diodes, polymer laser, organic solar cells.

\section{INTRODUCTION}

In the past decade, the phenomena of surface plasmon resonance (SPR) have been extensively used to investigate optical constants and thickness of thin films, surface properties [1], dye doped polymer on metal film mediated by SPPs enhanced emission energy transfer [2-8], and molecular interactions on the solid-liquid interface [9-13]. The SPR is a charge-density oscillation that may exist at the interface of two media with dielectric constants of opposite signs, for instance, a metal and a dielectric. This phenomenon was first observed in metal grating in the early 1900s. Kretschmann used a metallic-film-coated $(\sim 50 \mathrm{~nm})$ prism to generate a SPR signal [11]. Since then, the Kretschmann prism-coupling device has been used extensively to study the optical properties of metallic thin films, including index of refraction $(n)$, extinction coefficient $(k)$, thickness $(d)$, and roughness [14]. Other than the traditional Kretschmann (attenuated total reflectance (ATR method)) and Otto (frustrated total reflectance (FTR)) configurations, several novel devices based on grating nanostructure of mixed hybrid configuration have been reported in literatures, and the other one calls the "LRSPR", we do this design by plating a spacer layer between the prism and metal layer, and it can excite the SP wave and LRSP wave by the same structure at the different incident angles $[15,16]$.

In the research report, we presented and discussed the physics of enhancing light emission efficiency from organic symmetric dielectric films on a lamellar grating nanostructure by using the coupled LSPPs. For our experiments, we prepared 1-D patterns of nanostructures by electron beam lithography and the grating line size is $100 \mathrm{~nm}$ and $400 \mathrm{~nm}$, with pitch size at $500 \mathrm{~nm}$ and $800 \mathrm{~nm}$. The resist thickness is $100 \mathrm{~nm}$ on silicon substrate with an exposure area of $1.2 \times 1.2 \mathrm{~mm}$. The PL measurements from these organic/metal grating coupler basaed on LRSPR will result in the changes of SPR spectrum. The relative intensity of the PL emission from the Air/Au LRSPR mode is far field greater than that of the Alq3/Au SPP and the latter of these is only seen at high polar angles.

"Contact author: Email: cwlinx@ntu.edu.tw ; Tel: +886-2-3366-527; Fax: +886-2-3366-5268

Plasmonics: Metallic Nanostructures and their Optical Properties IV, edited by Mark I. Stockman, Proc. of SPIE Vol. 6323, 63231V, (2006) · 0277-786X/06/\$15 - doi: 10.1117/12.679035 


\section{PRINCIPLE}

\subsection{SPR and LRSPR}

The traditional Kretschmann (attenuated total reflectance (ATR method)) and Otto (frustrated total reflectance (FTR)) configurations are used for SPR excitation. The SPR phenomenon occurs when the momentum of the TM light wave equals that of the SPW propagating between the metal and dielectric interface. Under these conditions, virtually all of the incident photon energy is transferred to the SPW $[1,15,16]$.

In the LRSPR configuration, the SPPs propagating associated with the upper and lower of the dielectric/metal/dielectric two bounding media interfaces. Each one of these two SPPs has an evanescent wave extending both into the dielectric medium and into the metal film. If the metal film is thick enough, the evanescent waves inside the metal that belong to the two SPW do not overlap as shown in Fig. 1(a). As the film thickness getting thinner, the evanescent waves of the otherwise decoupled modes begin to overlap as shown in Fig. 1(b), a transverse standing wave can be established. The degenerated SPP modes can possibly split into symmetrical or asymmetrical mode. Especially for the thickness of the thin metal film is in the range of 10 to $30 \mathrm{~nm}$, less energy dissipation in the metal with symmetric mode of "LRSPR", which leads to an increase of propagation length $[15,16]$.

a.

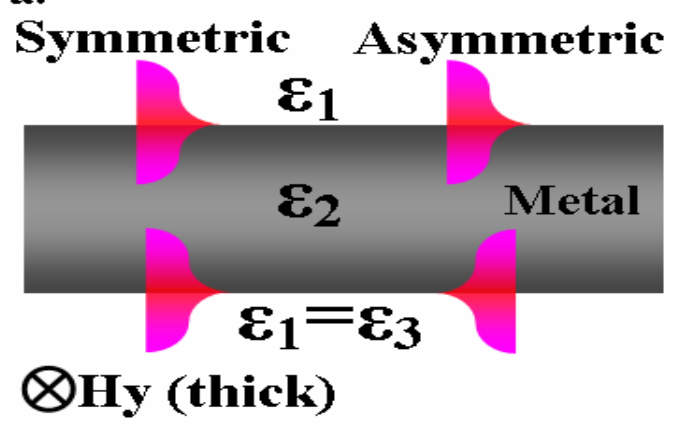

b.

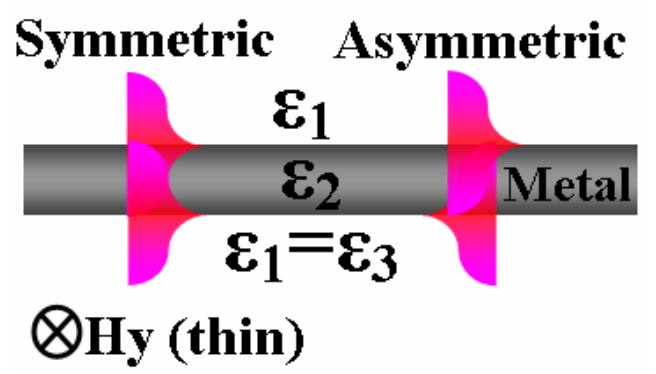

Fig. 1. Field distributions associated with bound, leaky, and growing modes guided by thickness metal film (a) and thin metal film (b). The arrows indicate energy flow in the dielectrics.

\subsection{Grating Excitation of a LRSPR}

A reflection grating is fabricated by a symmetrical lamellar reflective surface with periodical corrugation. An incident light with single-frequency planar polarization will spread into different orders, upon reflection,. The reflective angle depends on the incident wavelength. We have develop a measuring system for the angles of the reflected waves produced by a grating. The angle of reflection of $m$ th order may be related to the angle of incidence,by Eq. (1).

$$
\sin \theta_{R}^{(m)}=\sin \theta_{i}+\frac{m \lambda}{\Lambda},
$$

where $\theta_{i}$ is angle of incidence, $\theta_{R}^{(m)}$ is angle of reflection of $m$ th order and depends on wavelength, when $\Lambda$ too large the $\theta_{R}^{(m)}=\theta_{i}$ or $\Lambda$ too small the $\theta_{R}^{(m)}$ becomes imaginary, therefore $k_{x}$ becomes surface evanescent wave in $\mathrm{x}$ axis propagation.

The grating coupler is based on light diffraction on a periodically modulated surface of a diffraction grating, the coupling condition can be expressed as Eq. (2) and (3), where the $\boldsymbol{\Lambda}$ is the pitch of the grating [17].

The arrangement of a symmetrical periodically lamellar layer dielectric constant $\varepsilon_{2}$ in between two ordinary dielectrics of dielectric constant $\varepsilon_{1}$ and $\varepsilon_{3}$ with a symmetrical lamellar reflective surface with periodical corrugation. Figure 2 is a sketch of field $(E)$ and surface charge distributions for the two standing wave solutions at the metal grating gap boundaries. The SPR dispersion relationship curve for SPW along grating coupler propagating on the Air/Au and Alq3/Au interface boundaries is shown in Fig. 3. [7-8]. 


$$
n_{a} \sin (\theta) \pm m \frac{\lambda}{\Lambda}= \pm \sqrt{\frac{\varepsilon_{m r} n_{a}^{2}}{\varepsilon_{m r}+n_{a}^{2}}}=k_{S P P}
$$

and

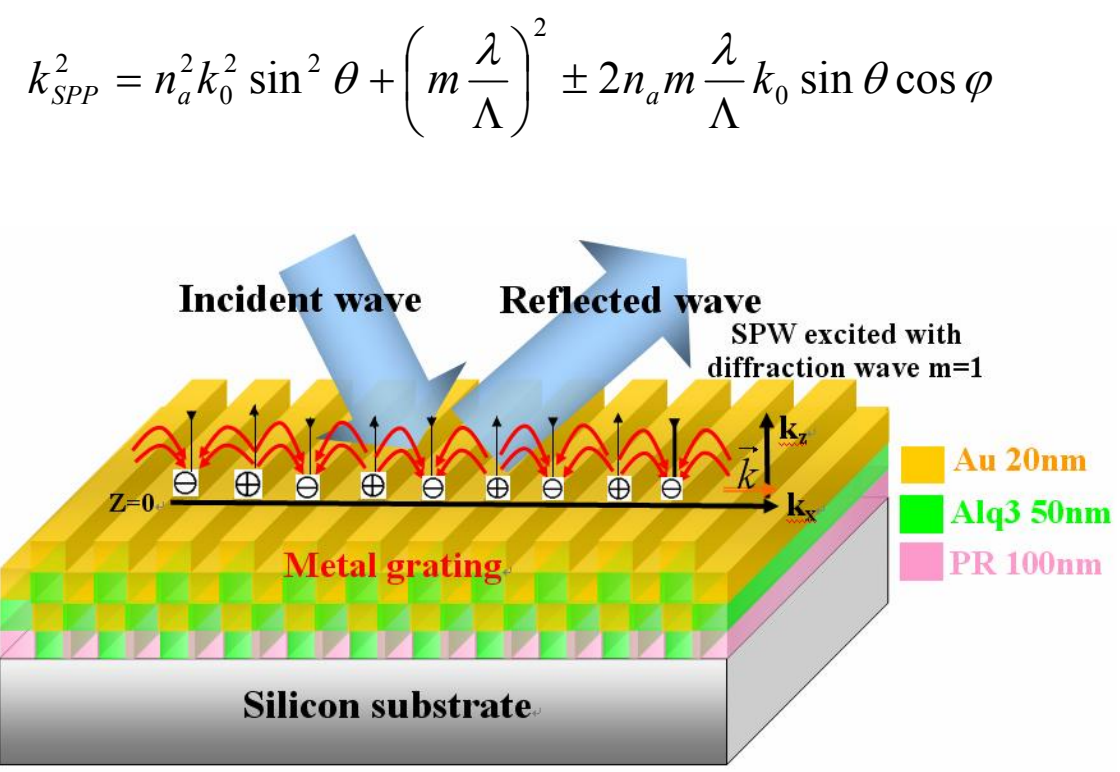

Fig. 2. Excitation of SP Bragg reflector on periodically modulated lamellar grating nanostructure with LRSPP propagation. The grating of symmetric mode is from $\mathrm{Au}(20 \mathrm{~nm}) / \mathrm{Alq} 3(50 \mathrm{~m}) / \mathrm{Au}(20 \mathrm{~nm}) / \mathrm{Alq} 3(50 \mathrm{~nm}) / \mathrm{PR}(100 \mathrm{~nm}) / \mathrm{Si}$ film layers and substrate.

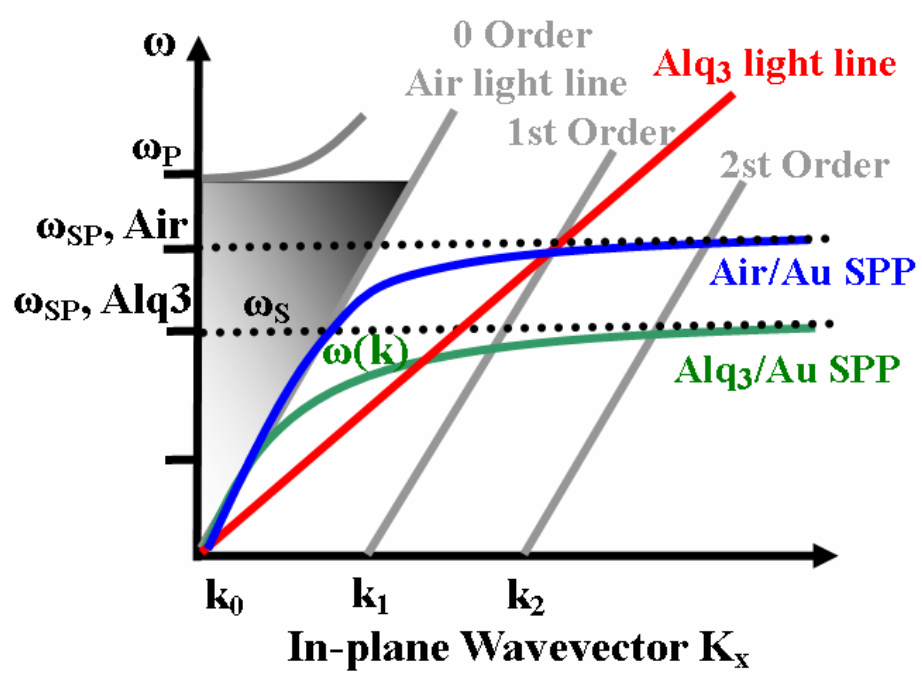

Fig. 3. Schematic representation of dispersion curves for surface plasmon polaritons propagating along oblique reflection on the grating surface of an Air/Au and Alq3/Au boundaries. 


\section{EXPERIMENTAL METHODS}

\subsection{Lamellar grating nanostructure fabrication}

The samples are prepared by an Electron Beam Lithography system (ELS-7500EX, Maker company).The incident electron beam with energy of $50 \mathrm{KV}$ was used in the experiment. In the first step, we used the electron-beam resist ZEP520A (To-Nippon Zeon Co.), which is a high resolution positive electron beam resist, to spin coating a resist layer on silicon substrate with $100 \mathrm{~nm}$ thickness, and then pre-bake at $180^{\circ} \mathrm{C}$ for 2 minutes. After exposure to the electron beam, the shape of the individual grating was composed of elongated one-dimensional line pattern of nanostructure with size of $100 \mathrm{~nm}$ and $400 \mathrm{~nm}$, and pitch of $500 \mathrm{~nm}$ and $800 \mathrm{~nm}$. The total exposure area is $1.2 \times 1.2 \mathrm{~mm}$ by pixel map of $60000 \times 60000$ dots and dose time of $2 \mu \mathrm{sec}$. Next, two pairs of $50 \mathrm{~nm}$ organic Alq3 film and $20 \mathrm{~nm}$ gold film was deposited by thermal evaporator with vacuum level at about $10^{-6}$ Torr onto the grating with an evaporation rate of approximately $0.5 \AA / \mathrm{s}$.

\subsection{Optical Set up}

The reflectivity measurement system for grating experiment was performed at room temperature using the setup as shown in Fig. 4. A white light source (Newport Oriel Spectral Luminator 69050) is used with a TM-polarization filter to illuminate the grating chip. The beam shape is controlled with a focus lens of large focal distance. The grating chip is placed at the center of a high resolution rotary angle $(\varphi)$ stage. The output beam is collected through a 2 inch lens with focal distance of $5 \mathrm{~cm}$ on a spectrometer (Ocean Optics Co.) or a CCD to measure output optical signal change in response to ambient refractive index. It resulted in the observable of the angle shift $(\theta)$ or intensity change in the reflectance.

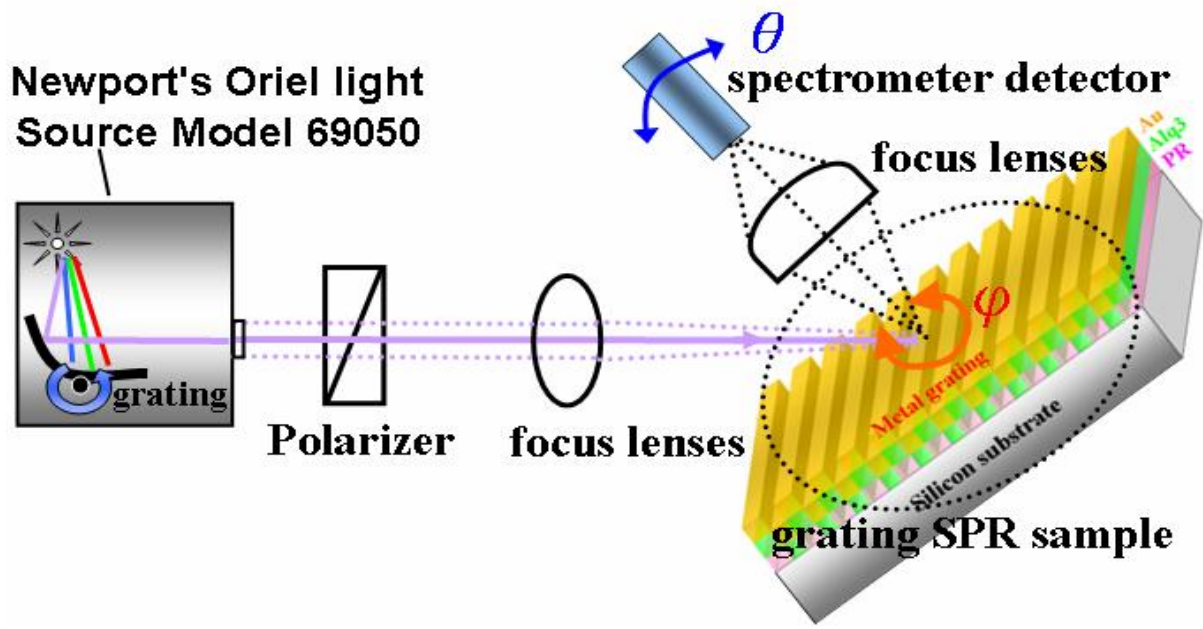

Fig. 4. Schematic sketch shows the experimental set-up for the PL spectra measurement. The sample geometry, the polar emission angle is $\theta$, the azimuthal angle is $\varphi$. A spectrometer is used to measure the emission from a white light source through the required energy range, whilst the sample is rotated in order to keep the in-plance momentum of the incident light constant throughout a particular sweep of energy.

For practical applications of grating LRSPR device, we have to apply Bragg equation to calculate the reflectance from a lamellar grating structure. Such a periodic metal nanostructure can have very interesting and exciting optical properties which strongly depend on the used materials, layer thickness, and grating pitch.

This can increase the amount of light emitted in the forward direction, and modifies the spectrum and spatial distribution of the emitted light. We examined the long-range SP mode from emission of organic molecules over a wavelength range of $450 \mathrm{~nm}$ to $750 \mathrm{~nm}$ and the results were compared both with Bragg diffraction theory for grating systems and with analytic expressions that describe the possible interacting modes in the thin-film limit. 


\section{RESULTS AND DISCUSSION}

\subsection{Lamellar grating nanostructure}

We used an EB resist mould with a pre-fabricated 1D line pattern of grating structure. Figure 5A and 5B show the top-view SEM (ELS-7500EX, ELIONIX CO.) images of patterned gold thin films. Their surface profiles measured with atomic-force microscopy (AFM) are shows in Fig. 6A and 6B. The grating profile calculated with the unified method is shown in the figure $6 \mathrm{C}$. The heights of the AFM profiles agree well with those of the calculated profiles.
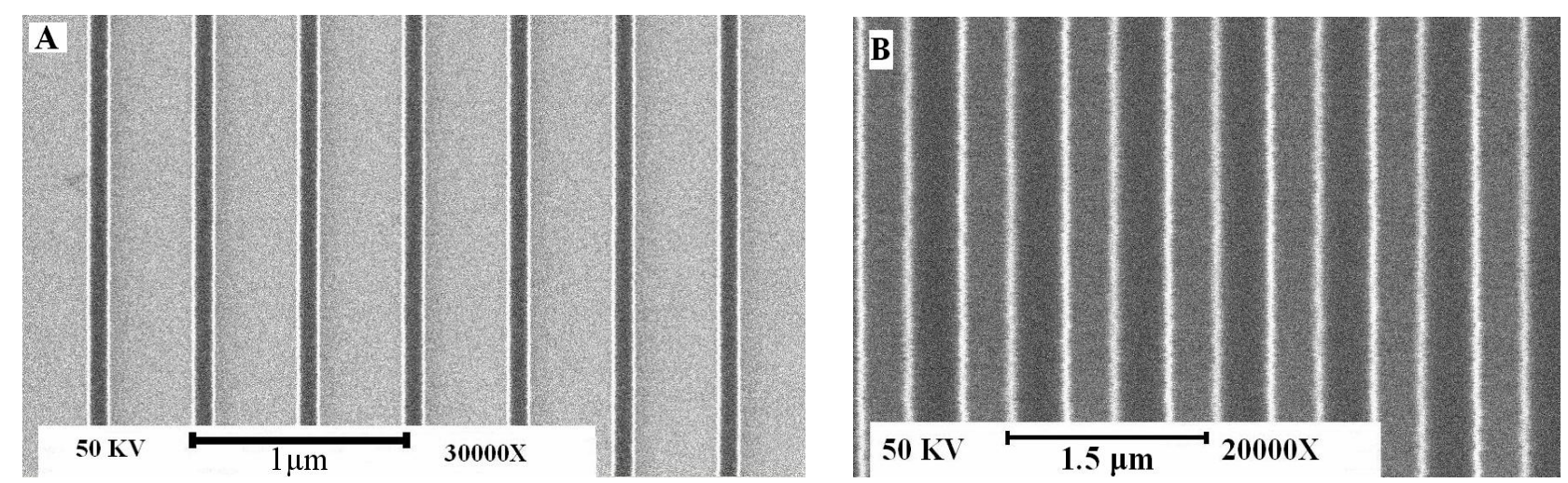

Fig. 5. The SEM images of the fabricated $100 \mathrm{~nm}$ thick resist period grating layer on silicon substrate for the (A) line size of $100 \mathrm{~nm}$, pitch of $500 \mathrm{~nm}$, and (B) line size of $400 \mathrm{~nm}$ and pitch of $800 \mathrm{~nm}$,..
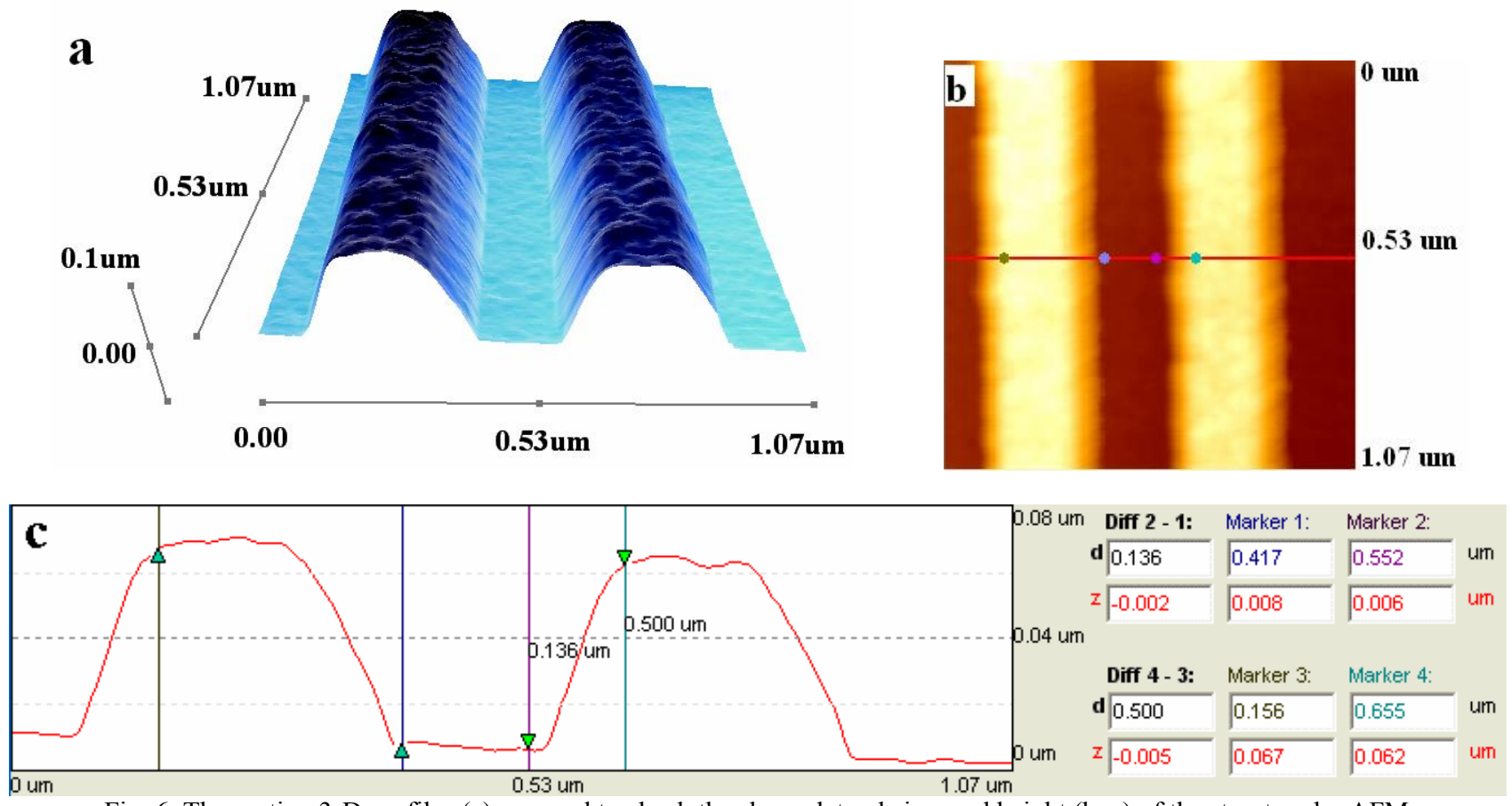

Fig. 6. The grating 3-D profiles (a) are used to check the shape, lateral size, and height (b, c) of the structure by AFM. 


\subsection{Emission characteristics}

We have examined the PL emission spectra through various fabricated structure layers to determine the emissive angle in LRSPR modes. The PL measurements on metal/organic grating, changes in SPR were measured from the angular spectrum as shows in Fig. 7. (grating $100 \mathrm{~nm}$, pitch 500nm) and Fig. 8. (grating 400nm, pitch 800nm). The angular spectra were cut-off due to the limitation of used spectrometer detector. We also examined the effect of surface plasmon excitation on the angular dispersion of emission wavelength. The resonance peaks for the coupling grating were obtained from rotary table measuring azimuthal angle $\varphi=0^{\circ}$. The emission spectra were measred at different angle $(\theta=$ $-25 \sim 25$ degree). The PL emission obtained from a grating sample containing Alq3/metal layer, excitation was provided by 410nm incident light (Oriel Spectral Luminator 69050), and the PL was detected by a spectrometer (Ocean Optics Co.)

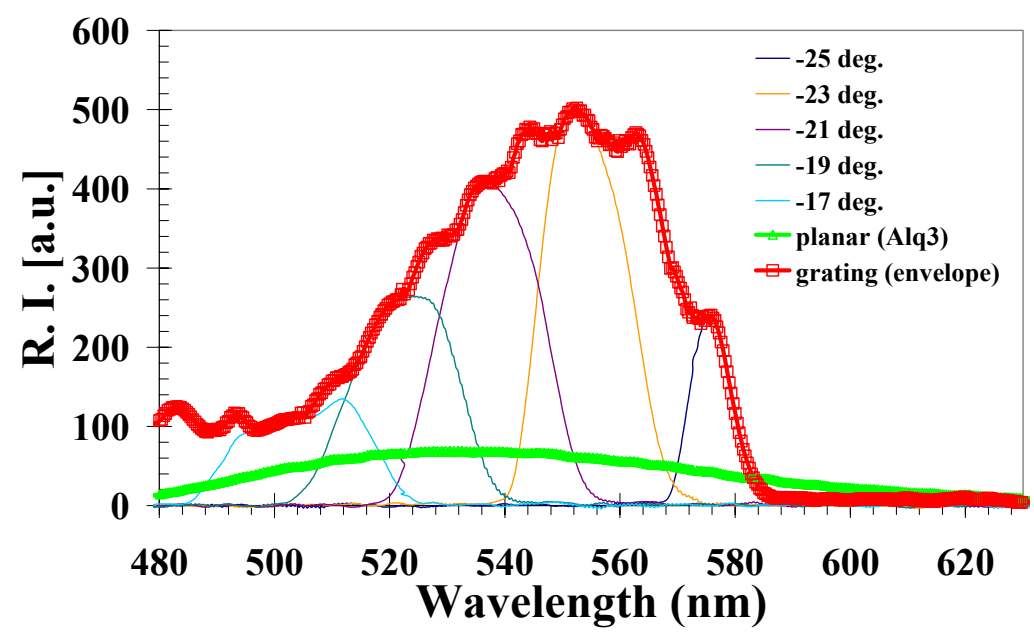

Fig. 7. The PL emission obtained from a grating sample containing Alq3 (80nm)/metal(40nm) layer, grating size (line $100 \mathrm{~nm}$, pitch $500 \mathrm{~nm}$, area size $1.2 \times 1.2 \mathrm{~mm}$ ). The figure shows the PL of grating and no grating (planar) sample of structure, and the integrated over all emission angles (envelope).

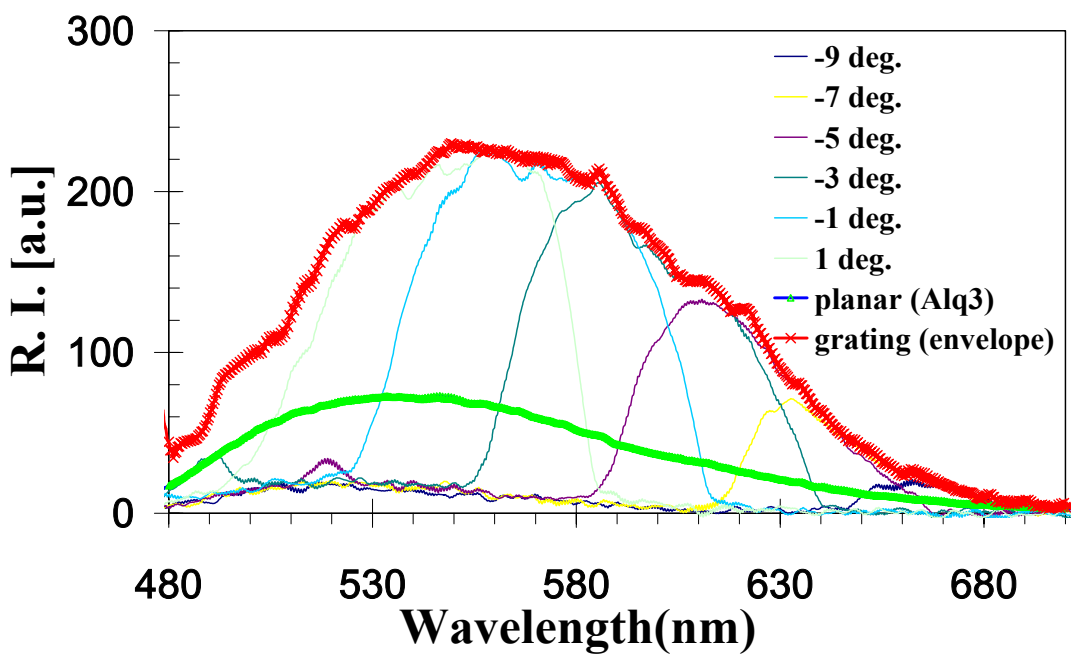

Fig. 8. The PL emission obtained from a grating sample containing Alq3 (80nm)/metal(40nm) layer, grating size (line 400nm, pitch $800 \mathrm{~nm}$, area size $1.2 \times 1.2 \mathrm{~mm}$ ). The figure shows the PL of grating and no grating (planar) sample of structure, and the integrated over all emission angles (envelope). 
The measurements of the PL emission in a plane perpendicular to the lines of the grating $100 \mathrm{~nm}$ and grating $400 \mathrm{~nm}$ are show in Fig. 9.

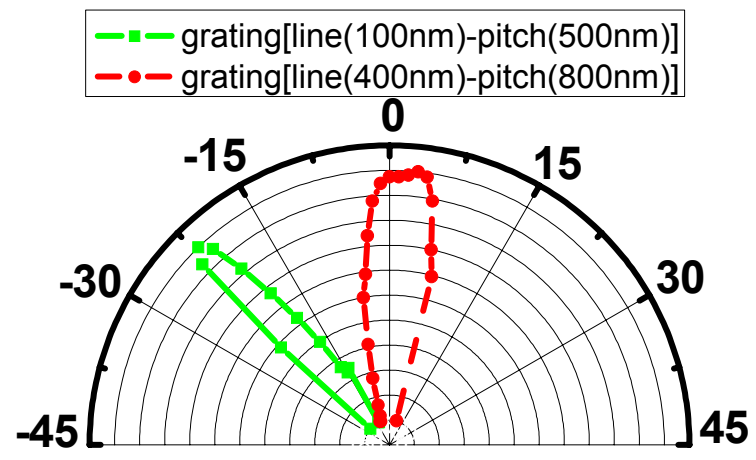

Fig. 9. Experimental PL emissivity diagram in polar coordinates of $(-\|-)$ grating $100 \mathrm{~nm}$, pitch $500 \mathrm{~nm}$ and $(-\bullet-)$ grating $400 \mathrm{~nm}$, pitch $800 \mathrm{~nm}$. To fit the data, we took into account the spectral resolution $2 \mathrm{~nm}$ and the angular resolution 1 degree.

\subsection{LRSPR Resonance angle measurements on organic/metal grating nanostructure}

We have measured the Au/Alq3 and Air/Au LRSPR radiation spectrum for each of our three samples by measuring the intensity of the grating components emitted in to their corresponding angles as determined by Eqs. (2) and (3). The data are presented for all three samples are shows in Fig.10, Fig. 11 and Fig. 12., beneath the 3D-images the intrinsic Alq3/Au emission to the Au/Air SP excitation spectrum.
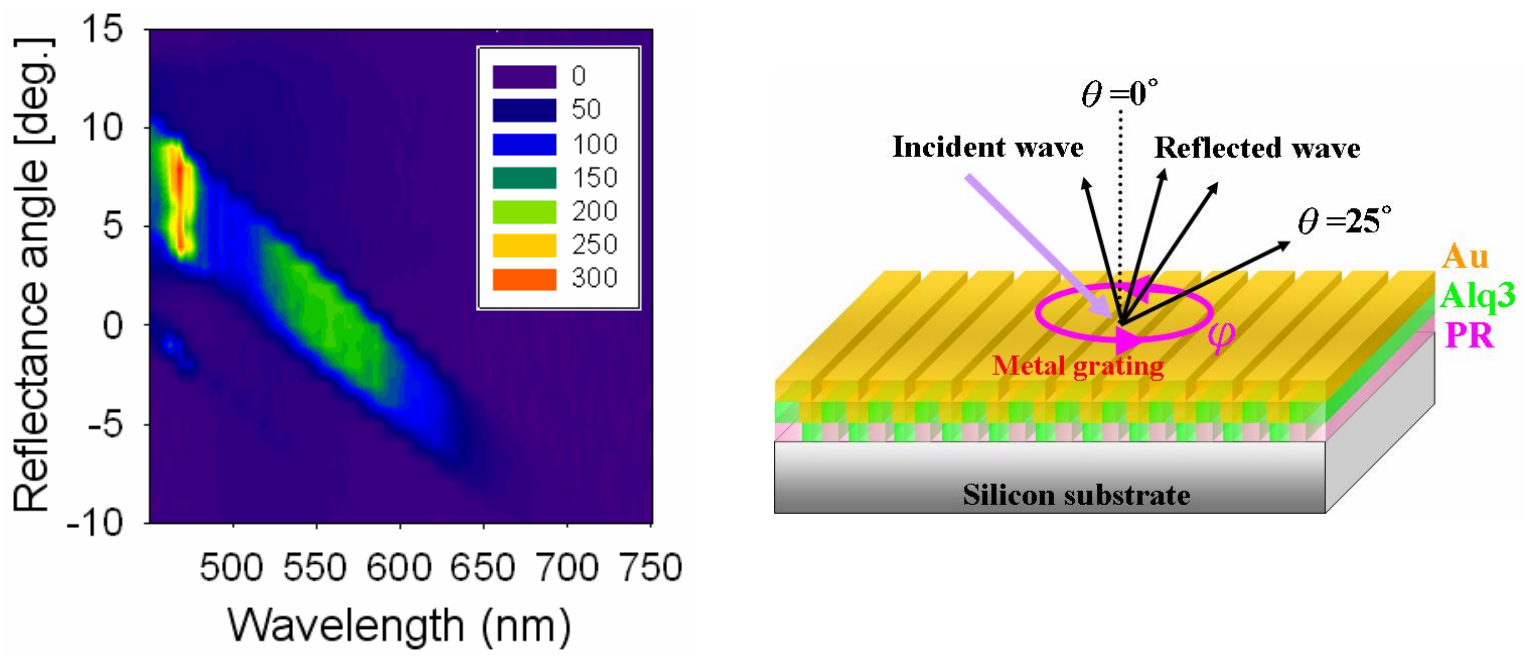

Fig. 10. The PL 3-D emission image (a) obtained from a grating sample containing Alq3/Au layer, grating size (line 400nm, pitch $800 \mathrm{~nm}$, area size $1.2 \times 1.2 \mathrm{~mm})$. PL measurements on $\mathrm{Si} /$ grating $(\mathrm{PR}) / \mathrm{Alq} 3(50 \mathrm{~nm}) / \mathrm{Au}(20 \mathrm{~nm})$ nanostructure show in (b), dependence of the emission spectra on the different angle $(\theta)$ of observation. The structure shows evidence of SPR mode on the Alq3/Au emission light lines to that the Air/Au interface resonance. (b) Shows the sample geometry, the polar emission angle is $\theta$, the azimuthal angle $\varphi=0$ degree. 

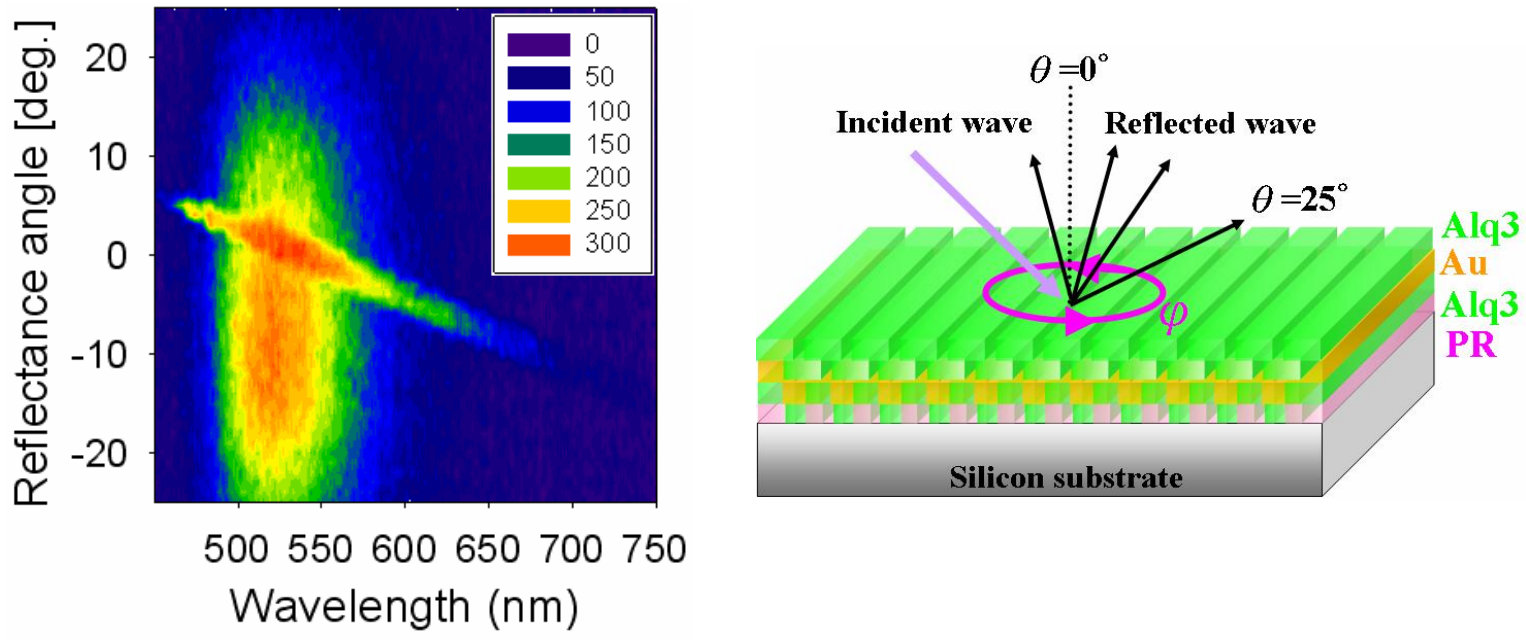

Fig. 11. The PL 3-D emission image (a) obtained from a grating sample containing Alq3/Au/Alq3 layer, grating size (line $400 \mathrm{~nm}$, pitch $800 \mathrm{~nm}$, area size $1.2 \times 1.2 \mathrm{~mm})$. PL measurements on $\mathrm{Si} /$ grating $(\mathrm{PR}) / \mathrm{Alq} 3(50 \mathrm{~nm}) / \mathrm{Au}(20 \mathrm{~nm}) / \mathrm{Alq} 3(50 \mathrm{~nm})$ nanostructure show in (b), dependence of the emission spectra on the different angle $(\theta)$ of observation. The structure shows evidence of SPR mode on the Alq3/Au and top layer Alq3 emission light lines to that the Air/Au interface resonance. (b) Shows the sample geometry, the polar emission angle is $\theta$, the azimuthal angle $\varphi=0$ degree.
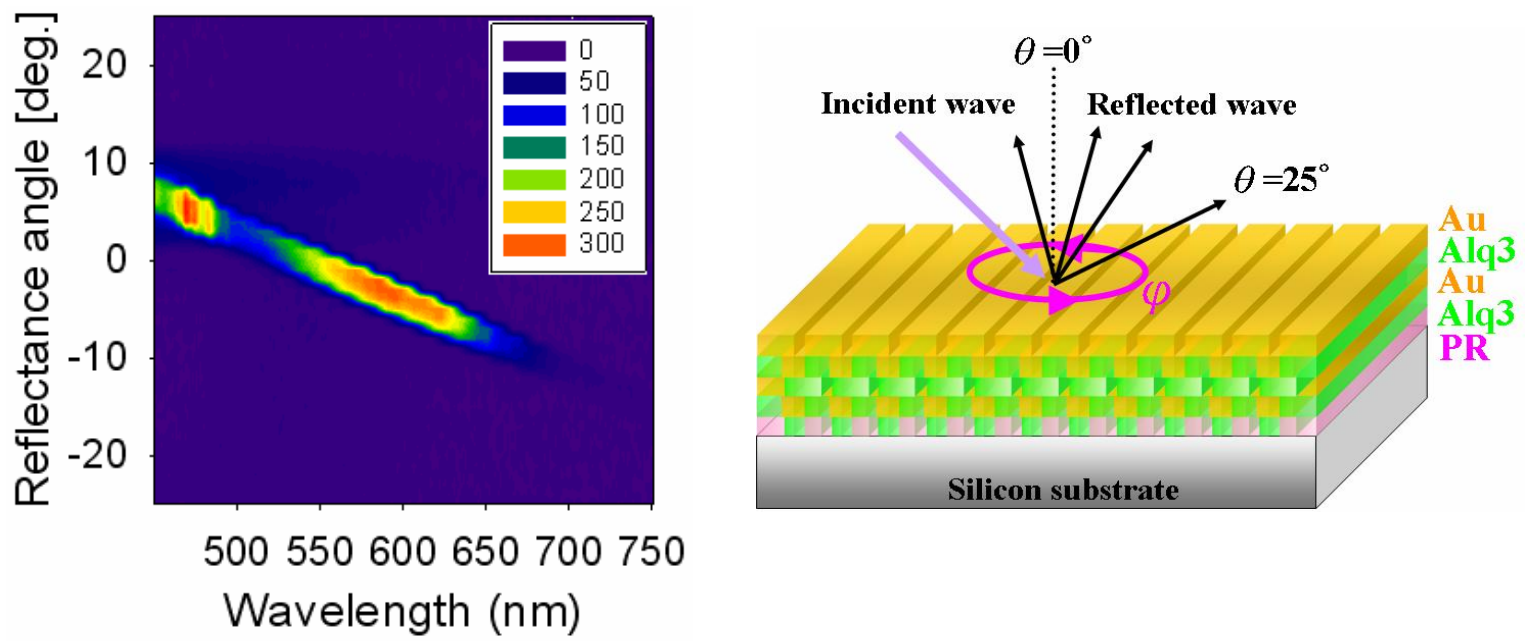

Fig. 12. The PL 3-D emission image (a) obtained from a grating sample containing Alq3/Au/Alq3/Au layer, grating size (line $400 \mathrm{~nm}$, pitch $800 \mathrm{~nm}$, area size $1.2 \times 1.2 \mathrm{~mm}) . \quad \mathrm{PL}$ measurements on Si /grating $(\mathrm{PR}) / \mathrm{Alq} 3(50 \mathrm{~nm}) / \mathrm{Au}(20 \mathrm{~nm}) / \mathrm{Alq} 3(50 \mathrm{~nm}) / \mathrm{Au}(20 \mathrm{~nm})$ nanostructure show in (b), dependence of the emission spectra on the different angle $(\theta)$ of observation. The structure shows evidence of SPR mode on the Alq3/Au and top layer Alq3/Au emission light lines to that the Air/Au interface resonance. (b) Shows the sample geometry, the polar emission angle is $\theta$, the azimuthal angle $\varphi=0$ degree. 


\section{CONCLUSIONS}

We have demonstrated guiding of long-range surface plasmon polaritons propagating along lamellar grating of 1-D rectangular lamellar grating in an organic/metal interface. Used to scatter SPP modes to light when such scattering takes place through a metal film have an important bearing on the effectiveness of the generation of useful light. We have shown experimentally that strong coupling between electronic and photonic resonances in LRSPR grating resonances in gold/Alq3 and Alq3/air symmetric mode leads to the formation of LRSPP devices. Therefore, further investigations are and will be performed of a LRSPP for integrated optical devices, examples of biosensor detectable or Organic light emitting diodes and organic solar cells applications of organic material/metal grating enhanced emission resonance energy interactions.

\section{ACKMOWLEDGEMENT}

This project is supported in part by National Science and Technology Program in Pharmaceuticals and Biotechnology, National Science Council, Taiwan, R.O.C., NSC 95-2323-B002-001, NSC 95-2323-B002-004, and MOEA 95-EC-17-A-05-S1-0017.

\section{REFERENCES}

1. H. Raether, Surface Plasmons on Smooth and Rough Surface and on Gratings (Springer-Verlag, Berlin, 1988).

2. $\quad$ P. Andrew, W.L. Barnes, "Energy transfer across a metal film mediated by surface plasmon polaritons", science, 306, 1002-1105 (2004).

3. T. D. Neal, K. Okamoto, A. Scherer, "Surface plasmon enhanced emission from dye doped polymer layers", Opt. Exp., 13, 5522-5527 (2005).

4. B. P. Rand, P. Peumans, S. R. Forrest, "Long-range absorption enhancement in organic tandem thin-film solar cells containing silver nanoclusters", J. Appl. Phys. 96, 7519-7526 (2004).

5. R. J. Crook, F. Yang, J. R. Sambles, Long-range optical modes supported by a strongly absorbing thin organic film, J. Opt. Soc. Am. B, 10, 237-243 (1993).

6. T. Nikolajsen, K. Leosson, I.Salakhutdinov, S. I. Bozhevolnyi, Polymer-based surface-plasmon-polariton stripe waveguides at telecommunication wavelengths, Appl. Phys. Lett. 82, 668-670 (2003).

7. S. Wedge, W. L. Barnes, "Surface plasmon-polariton mediated light emission through thin metal films", Opt. Exp. 12, 3673-3685 (2004).

8. S. Wedge, J. A .E. Wasey, I. Sage, W. L. Barnes, "Surface plasmon mediated emission from organic materials" SPIE proceeding. 4642, 79-87 (2002).

9. A. Otto, Excitation of nonradiative surface plasma waves in silver by the method of frustrated total reflection, $\mathrm{Z}$. Phys. 216, 398 (1968).

10. E. Kretschmann, Die Bestimmung optischer Konstanten von Metallen durch Anregung von Oberfldchenplasmaschwingungen, Z. Phys. 241, 313 (1971).

11. Z. Salamon, H.A. Macleod, G. Tollin, "Surface plasmon resonance spectroscopy as a tool for investigating the biochemical and biophysical properties of membrane protein system. I. Theoretical principles", Biochim. Biophys. Acta 1331, 117-129 (1997).

12. Z. Salamon, H.A. Macleod, G. Tollin, "Surface plasmon resonance spectroscopy as a tool for investigating the biochemical and biophysical properties of membrane protein system". II. Applications to biological systems", Biochim. Biophys. Acta 1331, 131-152 (1997).

13. J. Homola, S.S. Yee, G. Gauglitz, "Surface plasmon resonance sensor: review", Sens. Actuators B 54 3-15 (1994).

14. W.P. Chen, J.M. Chen, "Use of surface plasma waves for determination of the thickness and optical constants of thin metallic films", J. Opt. Soc. Am. 71, 189-191 (1981).

15. F.Y. Kou, T. Tamir, "Range extension of surface plasmons by dielectric layers", Opt. Lett. 12, 367-369 (1987).

16. D. Sarid, "Long-range surface-plasma waves on very thin metal film", Phys. Rev. Lett. 47, 1927-1930 (1981).

17. A. P. Hibbins, J. R. Sambles, "Azimuth-angle-dependent reflectivity data from metallic gratings", J. Opt. Mod. 45, p.1019-1028, 1998. 